\title{
Topological Graphene plasmons in a plasmonic realization of the Su-Schrieffer-Heeger Model
}

\author{
Tatiana G. Rappoport, ${ }^{1,2}$ Yuliy V. Bludov, ${ }^{3}$ Frank H. L. Koppens, ${ }^{4,5}$ and Nuno M. R. Peres ${ }^{3,6}$ \\ ${ }^{1}$ Instituto de Telecomunicações, Instituto Superior Técnico, \\ University of Lisbon, Avenida Rovisco Pais 1, Lisboa, 1049001 Portugal \\ ${ }^{2}$ Instituto de Fúsica, Universidade Federal do Rio de Janeiro, \\ Caixa Postal 68528, 21941-972 Rio de Janeiro RJ, Brazil \\ ${ }^{3}$ Department and Centre of Physics, and QuantaLab, \\ University of Minho, Campus of Gualtar, 4710-05\%, Braga, Portugal \\ ${ }^{4}$ ICFO-Institut de Ciencies Fotoniques, The Barcelona Institute of \\ Science and Technology, 08860 Castelldefels (Barcelona), Spain \\ ${ }^{5}$ ICREA-Institució Catalana de Recerca $i$ Estudis Avançats, Barcelona, Spain \\ ${ }^{6}$ International Iberian Nanotechnology Laboratory (INL), \\ Av. Mestre José Veiga, 4715-330, Braga, Portugal
}

(Dated: February 23, 2021)

\begin{abstract}
Graphene hybrids, made of thin insulators, graphene, and metals can support propagating acoustic plasmons (AGPs). The metal screening modifies the dispersion relation of usual graphene plasmons leading to slowly propagating plasmons, with record confinement of electromagnetic radiation. Here, we show that a graphene monolayer, covered by a thin dielectric material and an array of metallic nanorods can be used as a robust platform to emulate the Su-Schrieffer-Heeger model. We calculate the Zak's phase of the different plasmonic bands to characterise their topology. The system shows bulk-edge correspondence: strongly localized interface states are generated in the domain walls separating arrays in different topological phases. We find signatures of the nontrivial phase which can directly be probed by far-field mid-IR radiation, hence allowing a direct experimental confirmation of graphene topological plasmons. The robust field enhancement, highly localized nature of the interface states, and their gate-tuned frequencies expand the capabilities of AGP-based devices.
\end{abstract}

Topology can lead to intriguing physical phenomena and it is at the heart of modern condensed matter physics [1-4]. It has been successfully extended to various classical wave systems, such as photonics [5]-7], acoustic [8] and mechanical systems [9]. It also has been playing an increasingly important role in nanophotononics [10, offering alternative ways to design novel optical devices [11.

In one dimension, the celebrated Su-Schrieffer-Heeger (SSH) is probably the simplest and most representative model with non-trivial topology [12, 13]. Originally, it describes electrons in a one-dimensional tight-binding model with staggered hopping amplitudes, defined as intracell and intercell hoppings [12. Depending of the ratio between the two hopping amplitudes, the chain can have two topologically distinct ground states. The variation of this ratio leads to a topological phase transition between the two phases, with the band gap closing and reopening. If the intercell hopping is stronger than the intracell hopping, the system is in a non-trivial topological phase. In this case, the bulk-edge correspondence [14] predicts the existence of end-states, and interface states when two lattices with different topological phases are connected [15].

Photonic and plasmonic systems provide a flexible platform for the SSH model [16 19. The effective intracell and intercell hoppings can be controlled, for example, by tuning distances via nanofabrication. Nontrivial topology in coupled plasmonic nanoparticle arrays has been previously realized in 1D plasmonic nanoparticle ar- rays 20 24]. These systems, similar to the Su-SchriefferHeeger model, exhibit highly localized edge states at their ends, which are robust against perturbations [25]. However, similarly to dielectric photonic crystals, it is difficult to dynamically tune the $1 \mathrm{D}$ plasmonic nanoparticle arrays and control their edge and interface states. To overcome these limitations, one possibility is the use of highly tuneable graphene plasmons [26, 27].

Graphene Plasmon-polaritons (GP) are vertically localized electromagnetic fields (that is, surface waves) that can be excited in both the mid-infrared (MIR) and the Terahertz $(\mathrm{THz})$ spectral ranges. They present oscillatory behavior at the interface between graphene and a dielectric 28]. They can exhibit high degree of spatial confinement when compared to a wavelength of the same frequency in free space [28 34]. The configuration of Metal-Insulator-Graphene (MIG) for GPs 28, 35 38 involving a thin insulating layer, can hold vertically confined modes with much larger momentum than normal graphene plasmons. In this limit, the dispersion relation of the GP becomes linear and the mode is known as acoustic graphene plasmon (AGP) [35, 37, 39]. With this hybrid system, records in the spatial confinement of electromagnetic radiation has been achieved [37, 40, 41].

AGPs in periodic systems, e.g. involving periodic metallic rods on graphene (separated by an insulator), form plasmonic bands and present a wealth of different physical effects [42]. The AGP's lateral confinement that originates from the metallic nanostructure mimics a plas- 
monic tight-binding model where graphene's gating and the distance between rods can control the effective hopping, modifying the band-structure and modulating the band gaps.

Here, we propose a novel one-dimensional topological graphene plasmonic crystal that consists of a monolayer graphene on top of a bulk substrate $S$ with permittivity $\varepsilon_{S}$, separated from a periodic structure of silver rods with cross-section of area $W^{2}=75 \times 75 \mathrm{~nm}^{2}$ by a thin dielectric spacer, of thickness $d=3 \mathrm{~nm}$ and permittivity $\varepsilon_{d}$ (see Fig 1 1 ). The main advantage of this structure is that it is based on a recent experimental setup to create graphene acoustic plasmons [37, and therefore is experimentally feasible. The extra ingredient consists in using two different separations between the rods, which can be easily fabricated with the same techniques. Furthermore, it avoids the use of metagates [26, 27].

As illustrated in Fig 1b, the 1D lattice unit cell contains two identical silver rods separated by a distance $a$ and symmetrically located with respect to the center of the unit cell. Neighboring rods from different unit cells are separated by a distance $b$. The periodic structure has a period $L=a+b+2 W$. As $a$ dictates the intracell effective hopping and $b$ is linked to the intercell effective hopping, it is convenient to define the ratio $f=(a-b) /(a+b)$ that controls the topology of our system. $f=0$ ( Fig. 17) implies the periodic structure studied previously [42] with a single effective hopping. Positive (negative) values of $f$ specifies that the intracell effective hopping of our $\mathrm{SSH}$ model is larger (smaller) than the intercell one, as shown in Fig 1 b.

We perform full-wave finite element frequency domain simulations 43 and semi-analytical plane-wave expansions to characterize our plasmonic SSH model (see Supplemetary Materials for the details [44]). For simplicity, graphene is simulated as a single layer with optical conductivity that is given by a Drude like expression $\sigma_{g}(\omega)=$ $4 \sigma_{0} E_{F} /(\pi(\hbar \gamma-i \hbar \omega))$ [28], where $\sigma_{0}=e^{2} / 2 \hbar, E_{F}$ is the Fermi energy, $\gamma$ is the relaxation rate and $\omega$ is the frequency of the incident light. Since we will be considering large graphene Fermi energies, finite temperature play no significant effect in our results. The frequency-dependent relative permittivities of $\mathrm{Ag}$ are taken from Ref. [45.

First, we analyse the eigenfrequencies and eigenmodes of the system and distinguish their topological phases for different values of $f$. We then proceed to describe the interaction of EM radiation with our MIG structure. We consider a $p$-polarized monochromatic plane-wave impinging on the array of metallic rods at normal incidence. We calculate the absorption spectra resulting from the coupling of AGPs with far-field radiation, which can be used to design and model experiments. In this context, we consider different setups: a periodic system, an interface of two semi-infinite arrays with different topological phases, and edge states of a semi-infinite array interfacing a perfect electric conductor (PEC) . Unless otherwise specified, $\epsilon_{S}=\epsilon_{d}=1, E_{F}=0.6 \mathrm{eV}$ and $\gamma=3 \mathrm{meV}$.

The dispersion of the plasmons in a periodic system with a single rod per unit cell of length $L / 2$ presents several plasmonic bands 42. If the same system is represented by a unit cell of length $L$ with two evenly located rods $(f=0)$, the dispersion can be depicted in a Brillouin zone $k=[0,2 \pi / L]$ which has half of the size of the original one. As a result of the band folding, the dispersions cross each other at $k=\pi / L$, which is a point of degeneracy. When calculating the plasmonic band structure, from the analogy with a simple one-dimensional tight-binding $\mathrm{SSH}$ model, we should expect a splitting of the original bands for $f \neq 0$ exactly at degenerate points of the band folding (that is, at $k=\pi / L$ ), with the size of the new gap being proportional to $|f|$. This can be observed in our band structure and loss function calculations: figure 17 shows a density plot of the loss function calculated with the plane-waves expansion with a superimposed band-structure for $f=1 / 15$. Each of the two original lowest bands for $f=0$ have one degenerate point at $k=\pi / L$, which leads to a band folding induced band gap, splitting the original bands in two. The yellow rectangle in Fig. 1 1 highlights one of these band splittings, which is very small, because of the value of $f$, but illustrates nevertheless the effect of the band folding. The new Bragg gaps for $f \neq 0$ are always located at $k= \pm \pi / L$. Fig. 1 1 presents the same data but for $f=2 / 3$ and one can see that the band folding induced gap increases for large values of $f$ and there are four well separated bands labeled from 1 to 4 . As expected, the band gap varies linearly with $|f|$ for small values of $f$ (see the supplementary material [44]), and the band structures for $\pm f$ are exactly the same, although they correspond to different topological phases.
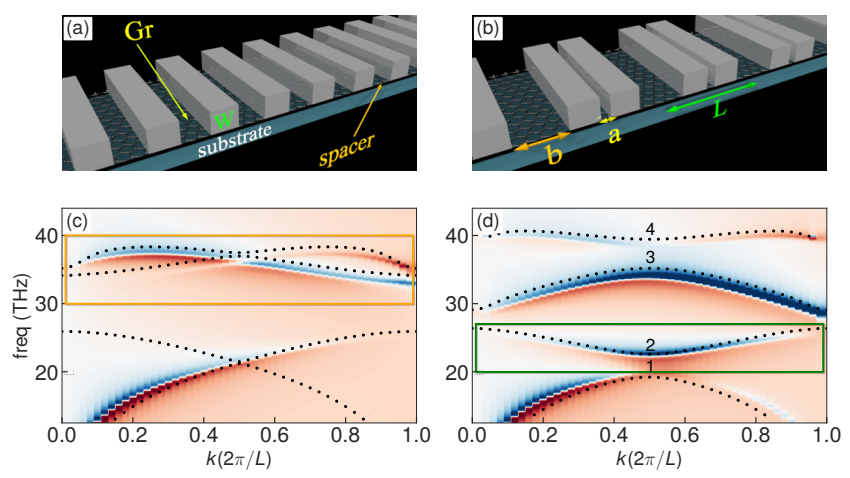

FIG. 1. (a)-(b) Illustration of the one dimensional array of rods with two rods per unit cell and $f=0$ and $f=2 / 3$ respectively. (c)-(d) Plasmonic band structure (dotted line) and loss function superimposed with the band structure (dotted lines), calculated for $\mathrm{f}=1 / 15$ (c) and $\mathrm{f}=2 / 3$ (d). The yellow rectangle in (c) highlights the band splitting at $k=\pi / L$ that occurs exactly at the degenerate point in the band folding for $f=0$. The numbers in panel (d) label the different bands

The 1D array has chiral symmetry, as the unit cell 
consists of two interconnected sublattices (one for each rod) that can be interchanged without modifying the system properties. One-dimensional periodical systems with chiral symmetry can be characterized by a topological invariant known as Zak phase [46. If the unit cell has an inversion symmetry, the Zak phase is quantized as $\pi$ (non-trivial) or 0 (trivial). To evaluate the Berry phase for electromagnetic waves in the absence of magneto-electric coupling, either the electric or magnetic fields can be considered in the calculation of the Berry connection $\vec{\Lambda}_{n, \vec{k}}$ [47]. We adopted the electric field in our calculations, where the permittivity tensor is isotropic and given by $\hat{\epsilon}(\vec{r})=\epsilon(\vec{r})$. After considering these simplifications, the Berry connection for an isolated band is given by [16, 48]:

$$
\vec{\Lambda}_{n, \vec{k}}^{E}=\mathrm{i} \int_{u . c} d \vec{r} \epsilon(\vec{r}) \vec{E}_{n, \vec{k}}^{*}(\vec{r}) \cdot \nabla_{\vec{k}} \vec{E}_{n, \vec{k}}(\vec{r})
$$

where $\vec{E}_{n, \vec{k}}(\vec{r})$ is the periodic-in-cell part of the normalized Bloch electric field eigenfunction of a state on the $n$th band with wave-vector wavevector $\vec{k}$.

The periodic structure has periodicity in $\hat{x}$ and the system is a one-dimensional plasmonic lattice. In this situation, the Zak phase [46] is defined as $\theta_{n}=\int_{-\pi / L}^{\pi / L} d k \Lambda_{n, k}$. The integral of the Berry connection over the $\mathrm{BZ}-\pi / L \leq$ $k<\pi / L$ can be approximated as a summation of the contributions of small segments. If the $\mathrm{BZ}$ is divided into $N$ segments where $k_{N+1}=k_{1}, e^{-\mathrm{i} \theta_{n}\left(k_{i}\right)} \approx 1-\mathrm{i} \theta_{n}\left(k_{i}\right)=$ $1-\mathrm{i} \Lambda_{n, k} \delta k$. As we are dealing with plasmons confined in the region between the metallic rods and the graphene sheet, without loss of generality, we can calculate the Zak phase at a fixed height $z_{0}$ located in the spacer with homogenous permittivity $\epsilon_{S}$. The Zak phase of this segment $\theta_{n}\left(k_{i}\right)$ for a band $n$ is given by

$$
e^{-\mathrm{i} \theta_{n}\left(k_{i}\right)}=\int_{u . c} d x \vec{E}_{n, \vec{k}_{i}}\left(x, z_{0}\right) \vec{E}_{n, \vec{k}_{i+1}}\left(x, z_{0}\right),
$$

where $\theta_{n}$ can be calculated in a gauge-invariant formalism as $\theta_{n}=-\operatorname{Im}\left[\log \left(\prod_{i=1}^{N} e^{-\mathrm{i} \theta_{n}\left(k_{i}\right)}\right)\right]$ [49].

Alternatively, $\theta_{n}$ can be obtained by inspecting the parity of the field profiles. If the symmetries of the eigenmode at $k=0$ and $k= \pm \pi / L$ are the same (different), the Zak phase of this band is quantized as $0(\pi)$. Following this procedure, we illustrate the differences in the parity of the field profiles for band 2, highlighted in green in Fig 1 1 . Figure $2 \mathrm{a}$, b presents the field profile $E^{x}{ }_{n, k}\left(x, z_{0}\right) k=0$ and $k=\pi / L$ respectively. $z_{0}$ is located in the middle of the spacer, between graphene and the rods. The phase of the eigenmode is fixed in such a way that $\vec{E}_{n, k=0}\left(x, z_{0}\right)$ is real. For $k=0$ (Fig. 2 ) the field profiles for $f= \pm 2 / 3$ are both even with respect to the inversion center of the unit cell, located at $x=0$. On the other hand, for $k=\pi$ (Fig. $2 \mathrm{~b}$ ) the profile is even for $f=-2 / 3$ and odd for $f=2 / 3$. When comparing Fig.
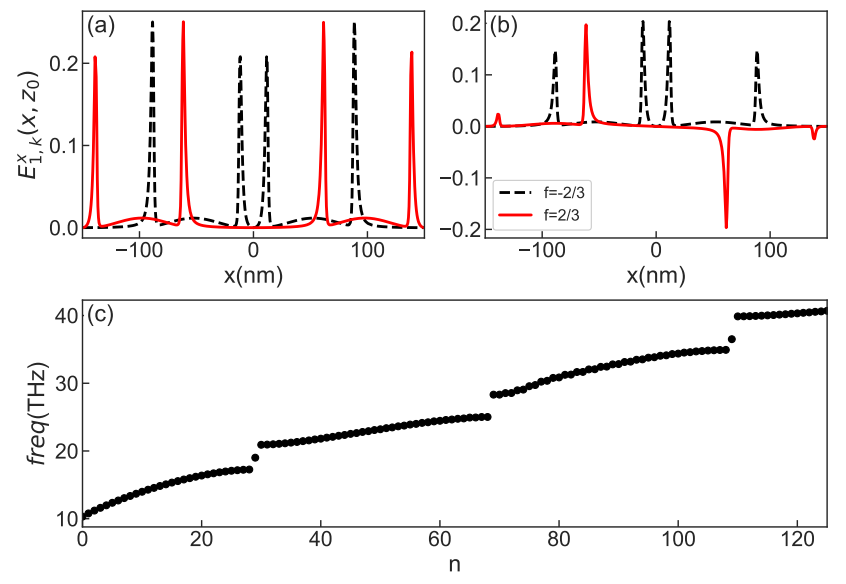

FIG. 2. The periodic part of the longitudinal component of the electric field $E_{n, \vec{k}}^{x}\left(x, z_{0}\right)$ for band 2, highlighted in Figure 11 for $k=0$ (a) and $k=\pi / L$ (b) where $z_{0}$ is located in the middle of the spacer $2.0 \mathrm{~nm}$ above graphene and $-L / 2 \leq x \leq$ $L / 2$. The dashed curves show the profiles for $f=-2 / 3$ while the profiles of the solid curves are calculated for $f=2 / 3$. (c) Energy spectrum of a composite system consisting of two connected finite arrays of 20 unit cells each with $f= \pm 2 / 3$ respectively, sandwiched by PECs. The mid-gap states are located in gaps after an odd number of bands.

$2 \mathrm{a}$ and $\mathrm{b}$ it is clear that for $f<0(f>0)$ the symmetries of the eigenmodes for $k=0$ (a) and $k=\pi / L(\mathrm{~b})$ are the same (opposite) so that $\theta_{n}=0(\pi)$, which corresponds to the value obtained by the Zak phase calculation following equation 2 .

Lets us now address the physical consequences and experimental signatures of the Zak phase in the graphene plasmonic crystal. To obtain a clear signature of the topology, one route is the observation of interface states for different values of $f$. Figure $2 \mathrm{c}$ shows the energy spectrum for a single finite system consisting of two neighbouring arrays of 20 unit cells each with $f= \pm 2 / 3$ respectively. The system is sandwiched by perfect electric conductors (PECs). As discussed previously, systems with the same $|f|$ have the same spectrum. Consequently, both arrays have the same band-structure and the spectrum of the four lowest bands for the composite system is similar to the unfolded version of the band structure of Fig 1 $\mathrm{d}$. However, there is a clear presence of midgap states inside the Bragg gaps. If two semi-infinite systems with different topological phases form an interface, the existence of a topological interface state in a given band gap is consistent with the bulk-edge correspondence. Thus, the mid-gap states of Figure 2 r are associated to the different Zak phases of each individual array with $f= \pm 2 / 3$, corroborating the previous analysis for periodic systems. The original bands for $f=0$ are split in two for $f \neq 0$ but they do not cross any other band, independent of the value of $f$. Because of the conservation of topological numbers in band theory, the sum 
of the Zak phases of each pair of these bands is always the same, regardless of the sign of $f$, although each individual band can change its phase when inverting the sign of $f$ and the Bragg gap goes to zero. This results in the absence of mid-gap states in gaps located after an even number of bands (see Fig 2p).

To explore the experimental signatures of the Zak phase, we consider the coupling of the plasmonic crystal with far-field radiation. In this case, we have a $p$ polarized monochromatic plane-wave impinging on the array of metallic rods at normal incidence. Let us first consider the periodic system and see if the splitting of the bands with different values of $f$ can be observed in far-field experiments. At normal incidence, TM modes couple with states with $k_{x} \sim 0$. Because of this, far-field experiments cannot directly obtain the linear dependence of the gap with $f$, as the gap opening associated to the SSH model occurs at $k=\pi / L$. Still, it is possible to capture: 1) the existence of an extra band for $f \neq 0$, and 2 ) the band separation at $k_{x}=0$ for increasing values of $|f|$. The first bands that can be seen in the far-field experiments with normal incidence, are bands 3 and 4, highlighted in Fig 1 c. This is illustrated in figure 3 a: the absorption spectra for $f=0$ has a single peak at this range of frequencies. For $f \neq 0$, the band is split in bands 3 and 4 and produces two peaks in the absorption spectra, where their position is dictated by the frequency of the bandstructure at $k=0$. The peak separation is not directly related to the band gap but, instead, to the values of the band structure for $k=0$.

To analyse the interface states, we begin by considering a finite plasmonic lattice with $f=-2 / 3$ with PEC ( perfect electric conductor) boundary conditions. This system presents exactly the same far-field response of the periodic lattice (see Fig. 3). We can now compare it with the response for the interface considered in Figure 2e, involving two joined arrays with $f= \pm 2 / 3$. In this case, the interface state between different topological phases of the two chain leads to an extra absorption peak located between the two original peaks of the infinite system, seen in Fig. 3b. Figure 3r shows the field enhancement at the interface state at this particular frequency. The dashed squares indicate the position of the metallic rods and the interface between the two different lattices is located at $x=0$. One can see that in the vicinity of the domain wall separating the two lattices, the field is enhanced in the whole space, including the region above the rods. Figure $3 d$ exhibits the profile of $|\vec{E}(\vec{r})| /\left|\vec{E}_{0}\right|$, at $z_{0}$, located in the spacer between graphene and the rods, where $\vec{E}_{0}$ is the electrical field. In this case, the field enhancement is of the order of 120-140, which is of the order of the field enhancement of the main absorption peaks 42. The electric field profile in Fig. 35 has a maxima that is strongly localized in the region of the interface between the two arrays. This differs from the extended electric field profile normally seen in the inter-
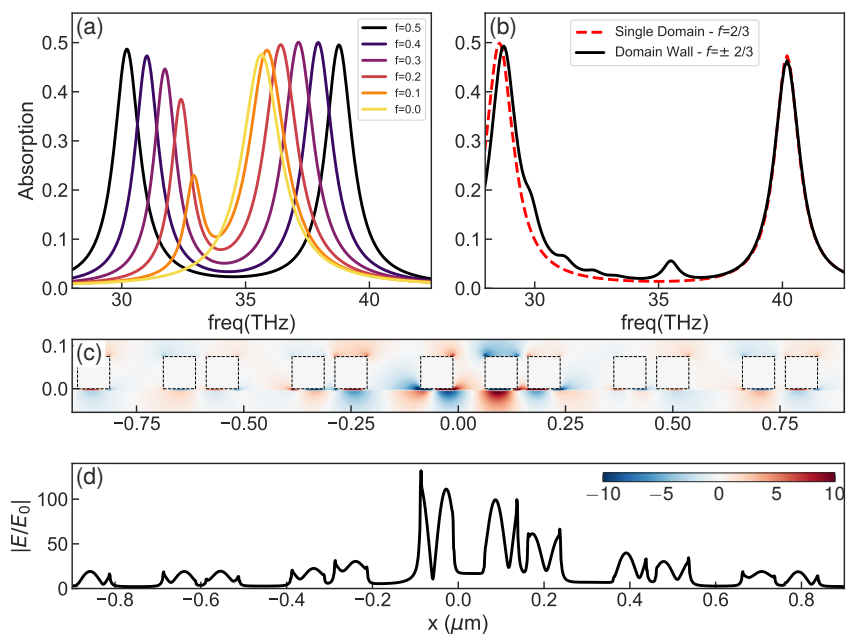

FIG. 3. (a) Absorption spectra for a periodic array and different values of $f=(a-b) /(a+b)$. (b) Absorption spectra of a finite plasmonic crystal with $f=-2 / 3$ PEC boundary conditions (dashed lines) and the interface containing two connected arrays of 6 unit cells each, with $f= \pm 2 / 3$ (solid line). (c) Electric field distribution $E^{y}(\vec{r}) / E_{0}$ for the region of the interface consisting of two connected arrays with $f= \pm 2 / 3$. The dashed squares denote the position of the metallic rods. (d) Electric field enhancement $|\vec{E}(\vec{r})| / E_{0}$ in the spacer between graphene and the rods. $x=0$ specifies the interface between the two arrays with $f= \pm 2 / 3$.

face of photonic crystals with different Zak phases, where the interface state has a width of several unit cells [50].

The topological nature of this peak can be further confirmed by comparing the absorption peaks of interfaces between two arrays with $f=f_{1}$ and $f=f_{2}$ where $\left|f_{1}\right| \neq$ $\left|f_{2}\right|$. In this case, one can produce interfaces between systems in the same topological phase $\left(\operatorname{sgn}\left(f_{1}\right)=\operatorname{sgn}\left(f_{2}\right)\right.$. However, only interfaces between systems with $\operatorname{sgn}\left(f_{1}\right) \neq$ $\operatorname{sgn}\left(f_{2}\right)$ produce interface states (see S. M). Although the topological nature of the bands cannot be easily tuned insitu, it is still possible to use a gate to modify the optical conductivity in graphene. This leads to a change in the size of the band widths and gaps and the exact frequency of the edge state. The flexibility to modify the frequency of the interface state can be useful for technological applications.

We can now consider a vacancy or a void in the unit-cell neighboring one of the PECs. This is obtained by removing the two silver rods belonging to the last unit cell of the right, as illustrated in Fig. 4p and c. In this case, the calculation of the eigenfrequencies of the finite system do not produce in-gap states that are related to the topology. Still, when analysing the interaction with far-field radiation, different responses emerge, depending on the Zak phase of the plasmonic band. For positive values of $f$, there is an extra peak in the absorption spectra (Fig. 4 a) which is related to an interface state and electric field enhancement of the electric field at the vacancy (Fig. $4 \mathrm{p}$ ). 
However, for negative $f_{\mathrm{s}}$, the absorption spectra is very similar to the periodic array and no interface states are observed. Instead, a resonator is formed between the last rod of the structure and the PEC. Depending on the size $D$ of the resonator, its eigenfrequency can be located inside the gap $(f>0)$ or in the plasmonic band $f<0)$, which explains the absorption spectra.

Although we cannot connect this response to topology, it can still be used in situations where one needs to produce a field confinement in the absence of the metallic rod, such as in sensing applications.

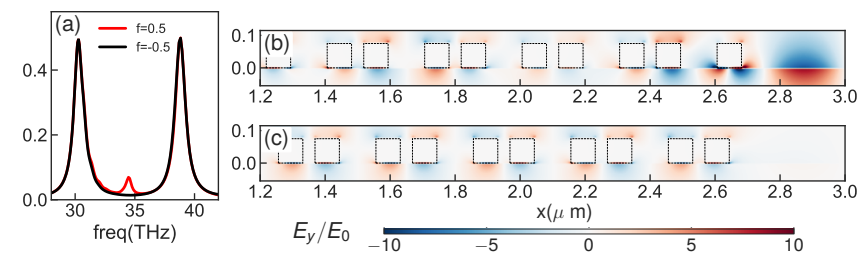

FIG. 4. (a) Absorption spectra for a plasmonic crystal with $f= \pm 1 / 2$ with one vacancy interfacing a perfect electric conductor. Electric field distribution $E^{y}(\vec{r}) / E_{0}$ for lattices with (b) $f=1 / 2$ and (c) $f=-1 / 2$ with one vacancy interfacing a perfect electric conductor. The dashed squares denote the position of the metallic rods.

Conclusions We proposed a simple structure to simulate the Su-Schrieffer-Heeger Model for plasmons in graphene, which avoids the use of meta-gating . Our setup is based on Metal-Insulator-Graphene systems that host acoustic graphene plasmons. Periodic arrays of metallic rods with two rods per unit cell generate plasmonic bands with topological properties that can be tuned by the distances between the rods. Interface states with strong field enhancement can be created at the interface between two arrays with different topologies. The frequency of the localized state can be easily tuned by gating graphene, which opens new avenues in plasmonic applications where light needs to be confined to a precise location in space or where a tunable narrow band absorption is needed.

TGR acknowledges funding from Fundação para a Ciência e a Tecnologia and Instituto de Telecomunicações - grant number UID/50008/2020 in the framework of the project Sym-Break and Mario G. Silveirinha for useful discussions. N.M.R.P. and F.H.L.K. acknowledge support from the European Commission through the project "Graphene-Driven Revolutions in ICT and Beyond" (Ref. No. 881603, CORE 3). N.M.R.P. acknowledge COMPETE 2020, PORTUGAL 2020, FEDER and the Portuguese Foundation for Science and Technology (FCT) through project POCI-01- 0145-FEDER-028114. F.H.L.K. acknowledges financial support from the Government of Catalonia trough the SGR grant, and from the Spanish Ministry of Economy and Competitiveness, through the "Severo Ochoa" Programme for Centres of
Excellence in RD (SEV-2015- 0522), support by Fundacio Cellex Barcelona, Generalitat de Catalunya through the CERCA program, and the Mineco grants Ramón y Cajal (RYC-2012-12281, Plan Nacional (FIS2013-47161$\mathrm{P}$ and FIS2014-59639- JIN) and the Agency for Management of University and Research Grants (AGAUR) 2017 SGR 1656. This work was supported by the ERC TOPONANOP under grant agreement n 726001 and the MINECO Plan Nacional Grant 2D-NANOTOP under reference no FIS2016-81044-P.

[1] M. Z. Hasan and C. L. Kane, Rev. Mod. Phys. 82, 3045 (2010).

[2] X.-L. Qi and S.-C. Zhang, Rev. Mod. Phys. 83, 1057 (2011)

[3] Y. Ren, Z. Qiao, and Q. Niu, Reports on Progress in Physics 79, 066501 (2016).

[4] J. Wang and S. Zhang, Nature Mater. 16, 1062 (2017).

[5] L. Lu, J. D. Joannopoulos, and M. Soljačić, Nature Photonics 8, 821 (2014)

[6] T. Ozawa, H. M. Price, A. Amo, N. Goldman, M. Hafezi, L. Lu, M. C. Rechtsman, D. Schuster, J. Simon, O. Zilberberg, and I. Carusotto, Rev. Mod. Phys. 91, 015006 (2019)

[7] M. G. Silveirinha, Phys. Rev. B 92, 125153 (2015).

[8] Z. Yang, F. Gao, X. Shi, X. Lin, Z. Gao, Y. Chong, and B. Zhang, Phys. Rev. Lett. 114, 114301 (2015).

[9] G. Ma, M. Xiao, and C. T. Chan, Nature Reviews Physics 1, 281 (2019)

[10] M. S. Rider, S. J. Palmer, S. R. Pocock, X. Xiao, P. Arroyo Huidobro, and V. Giannini, Journal of Applied Physics 125 (2019), 10.1063/1.5086433, arXiv:1812.08679

[11] Y. Ota, K. Takata, T. Ozawa, A. Amo, Z. Jia, B. Kante, M. Notomi, Y. Arakawa, and S. Iwamoto, Nanophotonics 9, $547(2020)$.

[12 W. P. Su, J. R. Schrieffer, and A. J. Heeger, Phys. Rev. Lett. 42, 1698 (1979).

113 W. P. Su, J. R. Schrieffer, and A. J. Heeger, Phys. Rev. B 22, 2099 (1980)

[14] J.-W. Rhim, J. Behrends, and J. H. Bardarson, Phys. Rev. B 95, 035421 (2017).

[15] A. J. Heeger, S. Kivelson, J. R. Schrieffer, and W. P. Su, Rev. Mod. Phys. 60, 781 (1988).

[16] M. Xiao, Z. Q. Zhang, and C. T. Chan, Phys. Rev. X 4, $021017(2014)$

[17] W. S. Gao, M. Xiao, C. T. Chan, and W. Y. Tam, Opt. Lett. 40, 5259 (2015).

[18] Q. Wang, M. Xiao, H. Liu, S. Zhu, and C. T. Chan, Phys. Rev. B 93, 041415 (2016).

[19] J. C. G. Henriques, T. G. Rappoport, Y. V. Bludov, M. I. Vasilevskiy, and N. M. R. Peres, Phys. Rev. A 101, 043811 (2020)

[20] C. W. Ling, M. Xiao, C. T. Chan, S. F. Yu, and K. H. Fung, Optics Express 23, 2021 (2015)

[21] C. A. Downing and G. Weick, Physical Review B 95 (2017), 10.1103/physrevb.95.125426.

[22] D. E. Gómez, Y. Hwang, J. Lin, T. J. Davis, and A. Roberts, ACS Photonics 4, 1607 (2017). 
[23] C. Li and A. Miroshnichenko, Physics 1, 2 (2018)

[24] S. R. Pocock, X. Xiao, P. A. Huidobro, and V. Giannini, ACS Photonics 5, 2271 (2018).

[25] S. R. Pocock, P. A. Huidobro, and V. Giannini, Nanophotonics 8, 1337 (2019).

[26] M. Jung, Z. Fan, and G. Shvets, Phys. Rev. Lett. 121, 086807 (2018)

[27] Z. Fan, S. Dutta-Gupta, R. Gladstone, S. Trendafilov, M. Bosch, M. Jung, G. R. Iyer, A. J. Giles, M. Shcherbakov, B. Feigelson, J. D. Caldwell, M. Allen, J. Allen, and G. Shvets, Nanophotonics 8, 1417 (2019).

[28] P. A. Gonçalves and N. M. Peres, An Introduction to Graphene Plasmonics (2016) pp. 1-431, arXiv:1609.04450.

[29] M. Jablan, H. Buljan, and M. Soljačić, Phys. Rev. B 80, $245435(2009)$

[30] F. J. G. De Abajo, ACS Photonics 1, 133 (2014), arXiv:1402.1969.

[31] H. R. Park, S. Namgung, X. Chen, N. C. Lindquist, V. Giannini, Y. Francescato, S. A. Maier, and S. H. Oh, Advanced Optical Materials 3, 667 (2015).

[32] F. H. Koppens, T. Mueller, P. Avouris, A. C. Ferrari, M. S. Vitiello, and M. Polini, Nature Nanotechnology 9, $780(2014)$

[33] T. Low and P. Avouris, ACS Nano 8, 1086 (2014), arXiv:1403.2799.

[34] U. Celano and N. MacCaferri, Nano Letters 19, 7549 (2019)

[35] P. Alonso-González, A. Y. Nikitin, Y. Gao, A. Woessner, M. B. Lundeberg, A. Principi, N. Forcellini, W. Yan, S. Vélez, A. J. Huber, K. Watanabe, T. Taniguchi, F. Casanova, L. E. Hueso, M. Polini, J. Hone, F. H. Koppens, and R. Hillenbrand, Nature Nanotechnology 12, 31 (2017), arXiv:1601.05753.

[36] M. B. Lundeberg, Y. Gao, R. Asgari, C. Tan, B. V. Duppen, M. Autore, P. Alonso-González, A. Woessner,
K. Watanabe, T. Taniguchi, R. Hillenbrand, J. Hone, M. Polini, and F. H. Koppens, Science 357, 187 (2017), arXiv:1704.05518

[37] D. A. Iranzo, S. Nanot, E. J. Dias, I. Epstein, C. Peng, D. K. Efetov, M. B. Lundeberg, R. Parret, J. Osmond, J. Y. Hong, J. Kong, D. R. Englund, N. M. Peres, and F. H. Koppens, Science 360, 291 (2018), arXiv:1804.01061

[38] P. A. D. Gonçalves, Plasmonics and Light-Matter Interactions in Two-Dimensional Materials and in Metal Nanostructures (Springer International Publishing, 2020).

[39] A. Principi, R. Asgari, and M. Polini, Solid State Communications 151, 1627 (2011)

[40] I. H. Lee, D. Yoo, P. Avouris, T. Low, and S. H. Oh, Nature Nanotechnology 14, 313 (2019)

[41] I. Epstein, D. Alcaraz, Z. Huang, V.-V. Pusapati, J.-P. Hugonin, A. Kumar, X. M. Deputy, T. Khodkov, T. G. Rappoport, J.-Y. Hong, N. M. R. Peres, J. Kong, D. R. Smith, and F. H. L. Koppens, Science 368, 1219 (2020).

[42] T. G. Rappoport, I. Epstein, F. H. L. Koppens, and N. M. R. Peres, ACS Photonics 7, 2302 (2020).

[43] "Comsol multiphysics(R) v. 5.4," (2018).

[44] "Supplementary material," (2020).

[45] M. G. Blaber, M. D. Arnold, and M. J. Ford, The Journal of Physical Chemistry C 113, 3041 (2009)

[46] J. Zak, Phys. Rev. Lett. 62, 2747 (1989).

[47] M. Onoda, S. Murakami, and N. Nagaosa, Phys. Rev. E 74, 066610 (2006).

[48] H.-X. Wang, G.-Y. Guo, and J.-H. Jiang, New J. Phys. 21, 093029 (2019).

[49] D. Vanderbilt, Berry Phases in Electronic Structure Theory (Cambridge University Press, 2018).

[50] Y. Meng, X. Wu, R.-Y. Zhang, X. Li, P. Hu, L. Ge, Y. Huang, H. Xiang, D. Han, S. Wang, and W. Wen, New Journal of Physics 20, 073032 (2018). 